\title{
An assessment of the invasive flora of Amchang Wildlife Sanctuary in the Kamrup District of Assam, India
}

\author{
Nandita Sarma ${ }^{1,2}$, Uma Shankar ${ }^{1,4}$ and Ashiho Asosii Mao ${ }^{3}$ \\ ${ }^{1}$ Department of Botany, North-Eastern Hill University, Shillong 793 022, Meghalaya, India \\ ${ }^{2}$ Botanical Survey of India, Eastern Regional Centre, Shillong 793 003, Meghalaya, India \\ ${ }^{3}$ Botanical Survey of India, C.G.O. Complex, Salt Lake City, Kolkata 700 064, West Bengal, India \\ ${ }^{4}$ Corresponding author: E-mail: arshuma@yahoo.com
}

[Received 01.12.2019; Revised \& Accepted 25.12.2019; Published 3.12.2019]

\begin{abstract}
Invasive alien species are not only harmful both ecologically and economically. but also pose a serious threat to the native flora risking species extinction. However, some invasive species are being used by the people for a variety of purposes like medicine, vegetables, furniture and for meeting their daily livelihood requirements. In this paper, we document a total of 57 invasive species belonging to 49 genera of 22 families with their local names in Assamese, frequency of occurrence, and flowering and fruiting periods from Amchang wildlife sanctuary in Assam. Of these, 24 species are medicinally used and seven species are cooked as vegetables. This information generated from the field work is a manifestation of the contemporary traditional knowledge of people about the plant resources growing around them in the sanctuary.
\end{abstract}

Key words: Amchang Wildlife Sanctuary, Assam, Invasive plants, medicinal use of plants.

\section{INTRODUCTION}

Invasion of natural ecosystems and replacement of native flora by the invasive alien plants is one among the top three threats identified globally by the scientists. India has also highlighted the adverse effects of invasion of flora in a number of studies and has set targets to reduce this menace in future decades (Bhatt et al. 2011). The northeastern region of India encompassing eight States, viz., (Arunachal Pradesh, Assam, Manipur, Meghalaya, Mizoram, Nagaland, Sikkim and Tripura) and northern districts of West Bengal (Darjeeling, Jalpaiguri and Coochbehar) is bestowed with a rich diversity of fauna, flora and microbes. This region constitutes the landscapes of two global hotspots of biodiversity, The Himalaya and IndoBurma (Myers 2003) and boasts a variety of ecosystems such as tropical lowland forests (Shankar \& Tripathi 2017; Shankar 2017, 2019), gregarious sal forests (Shankar 2001; Majumdar et al. 2014; Tripathi \& Shankar 2014), subtropical pine and oak forests (Upadhaya 2015), temperate conifer forests and savanna grasslands (Shankar 1991; Shankar et al. 1991). Orchidaceae is the largest family of flowering plants in this region and other dominant families are Poaceae, Compositae, Fabaceae, Rubiaceae, and Lamiaceae. The invasion of alien species in the northeastern region of India has emerged as a challenge for the $21^{\text {st }}$ century, despite the fact that its extent has not been quantified and it has not been subjected to scientific study (Shankar et al. 2011). Of late, a few studies on invasive plants were undertaken by some workers (Das 2002; Boro \& Sarma 2013; Buragohai 2007; Das \& Duarah 2013). Recently, a project has been launched by the Ministry of Environment, Forests, and Climate Change, Government of India under the aegis of National Mission on 
Himalayan Studies (NMHS) to study and document invasion of habitats in the Himalayan and northeastern region of India. Here, we present the results of a study carried out in the Amchang Wildlife Sanctuary in Kamrup district of Assam to document the diversity of invasive alien plants in the native habitats. We also investigated contemporary traditional knowledge of the people about the medicinal and economic uses of the invasive plants inhabiting in and around the sanctuary.

\section{MATERIALS AND METHODS}

Amchang Wildlife Sanctuary in Kamrup district of Assam comprises three reserve forests, viz., Amchang RF (53.18 km²), South Amchang RF (15.5 km²) and Khanapara RF (9.96 $\mathrm{km}^{2}$ ). Amchang was declared a sanctuary in 2004 with a total area of $78.64 \mathrm{~km}^{2}$. This sanctuary lies between $26^{\circ} 06^{\prime}$ to $26^{\circ} 13^{\prime} \mathrm{N}$ latitude and $91^{\circ} 50^{\prime}$ to $91^{\circ} 58^{\prime} \mathrm{E}$ longitude. Geographically, the area is a continuation of the Khasi and Jaintia hills of Meghalaya and forms a part of the Shillong Plateau (Shankar 2019). The Sanctuary is bounded by the river Brahmaputra in the North, by National Highway 37 and Sonapur in the South, by urban agglomeration of Guwahati city in the West and by Digaru Railway Station in the East (Figure 1). The ethnic groups such as Boro, Karbi, Kasari, Garo, Nepalese, Tea tribes and Assamese are residing in the fringe area of the Amchang Wildlife Sanctuary. These local people not only extract many wild plants and other non-timber forest products from the sanctuary as medicine and vegetables for their daily livelihood requirements, but also interact with vegetation in many other ways which paves the way for the spread of the invasive plants.

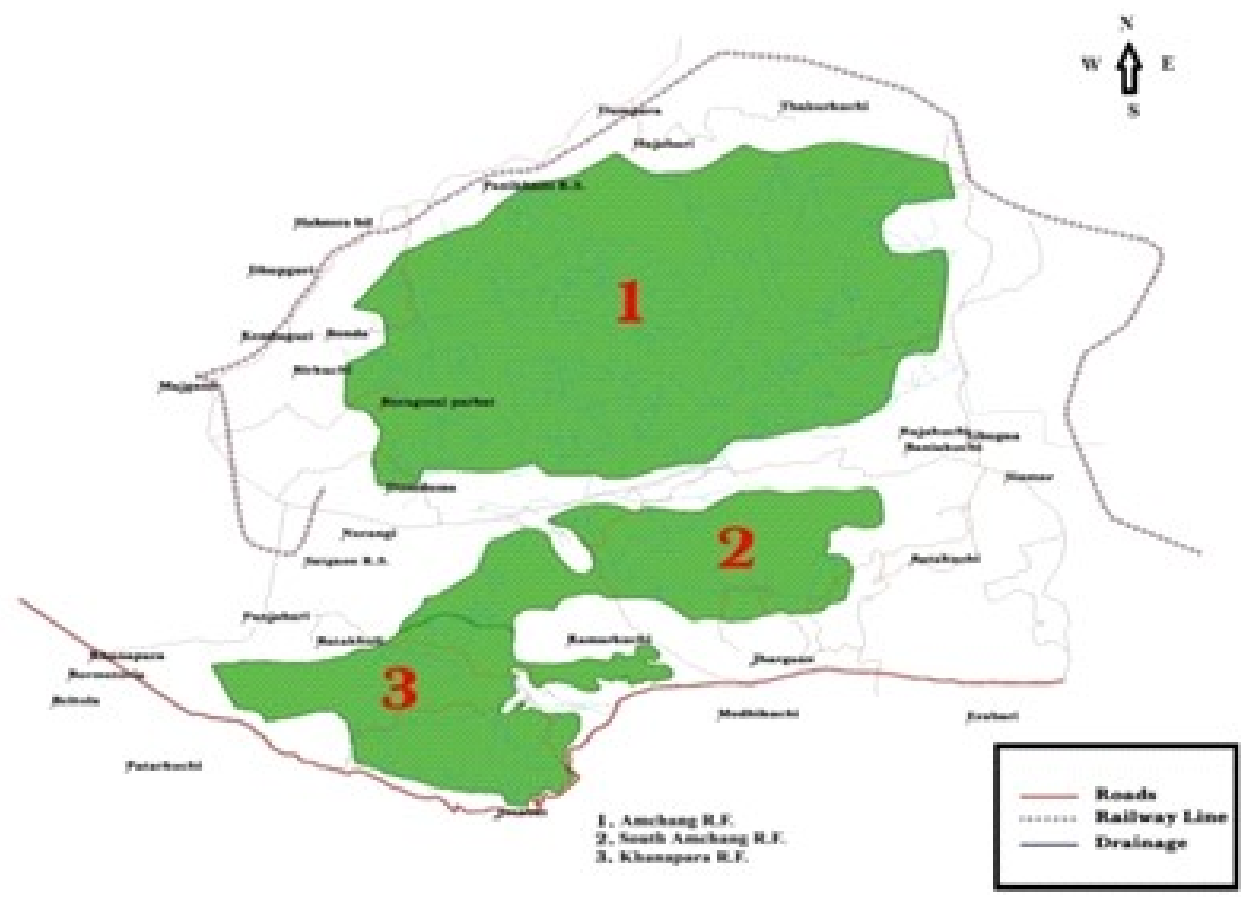

Figure 1. Map showing the location of Amchang Wildlife Sanctuary (source: Anonymous 2019). 
Several rounds of field visits to various parts of the forest areas in Amchang wildlife sanctuary, Assam (North-East India) were carried out in different seasons of the year during 2014-2019. During the surveys, live specimens along with photographs were taken. Plant species were collected and herbarium specimens were prepared (Jain \& Rao 1977) and preserved in laboratory for further investigation. The collected specimens were processed, mounted and identified with the aid of available resources (Barooah \& Ahmed 2014; Hooker 1872 - 1897; Kanjilal et al. 1934, 1936, 1938, 1940; The Plant List 2013) in the BSI herbarium and the NEHU library. The list of invasive species has been prepared following nomenclature of WFO (2019). The nativity of the exotic plants was recorded from the published literature (Das 2002; Boro et al. 2013; Khuroo et al. 2007; Reddy et al. 2008; Sekar 2012). A list of invasive species is tabled with scientific names, local names, habit and nativity.

\section{RESULTS AND DISCUSSION}

The field survey carried out in the Amchang Wildlife Sanctuary revealed that a total of 57 invasive species belonging to 49 genera and 22 families of angiosperms constituted the flora of the Sanctuary. Of these, the dicots were represented by 50 species belonging to 43 genera of 19 families and the monocots were represented by seven species belonging to six genera of three families (Table 1). The most dominant family was Compositae with 11 species followed by Solanaceae with six species, Fabaceae with five species, Amaranthaceae with four species and Cleomaceae, Convolvulaceae, Malvaceae and Poaceae with three species each (Table 1). The invasive species recorded from Amchang Wildlife Sanctuary were characterized by prolonged flowering and fruiting periods and some species flowered and fruited throughout the year. The local names of the species as spoken today are given as per the local dialect of the Assamese language. These names are colloquial and may differ from their literary utterances.

Table 1. The diversity of invasive plants in Amchang Wildlife Sanctuary.

\begin{tabular}{|c|c|c|c|c|c|}
\hline $\begin{array}{c}\text { Species name with collection } \\
\text { number }\end{array}$ & $\begin{array}{l}\text { Flowers } \\
\text { \& fruits }\end{array}$ & $\begin{array}{l}\text { Local name in } \\
\text { Assamese }\end{array}$ & Nativity & Habit & Occurrence \\
\hline $\begin{array}{l}\text { Ageratum conyzoides (L.) L. } \\
\text { [Compositae]; N. Sarma } 133898 \\
\text { (ASSAM) }\end{array}$ & $\begin{array}{l}\text { July - } \\
\text { January }\end{array}$ & $\begin{array}{l}\text { Gondhowa- } \\
\text { bon }\end{array}$ & $\begin{array}{l}\text { Tropical } \\
\text { America }\end{array}$ & Herb & Dominant \\
\hline $\begin{array}{l}\text { Alternanthera philoxeroides } \\
\text { (Mart.) Griseb.[Amaranthaceae]; } \\
\text { N. Sarma } 133866 \text { (ASSAM) }\end{array}$ & $\begin{array}{l}\text { August - } \\
\text { November }\end{array}$ & Pani-khutura & $\begin{array}{l}\text { Tropical } \\
\text { America }\end{array}$ & Herb & Dominant \\
\hline $\begin{array}{l}\text { Alternanthera sessilis (L.) R.Br. } \\
\text { ex DC. [Amaranthaceae]; } N . \\
\text { Sarma } 133981 \text { (ASSAM) }\end{array}$ & $\begin{array}{l}\text { July - } \\
\text { January }\end{array}$ & Mat-kaduri & $\begin{array}{l}\text { Tropical } \\
\text { America }\end{array}$ & Herb & Dominant \\
\hline $\begin{array}{l}\text { Amaranthus spinosus L. } \\
\text { [Amaranthaceae]; N. Sarma } \\
134015 \text { (ASSAM) }\end{array}$ & $\begin{array}{l}\text { July - } \\
\text { January }\end{array}$ & Kata-khutura & America & Herb & Frequent \\
\hline $\begin{array}{l}\text { Argemone mexicana L. } \\
\text { [Papaveraceae]; N. Sarma } \\
136580 \text { (ASSAM) }\end{array}$ & $\begin{array}{l}\text { September } \\
\text { - January }\end{array}$ & Sealkata & $\begin{array}{l}\text { Central \& } \\
\text { South } \\
\text { America }\end{array}$ & Herb & Occasional \\
\hline $\begin{array}{l}\text { Bidens pilosa L. [Compositae]; } \\
\text { N. Sarma } 136516 \text { (ASSAM) }\end{array}$ & $\begin{array}{l}\text { July - } \\
\text { December }\end{array}$ & $\begin{array}{l}\text { Deumeubai } \\
\text { (Bodo) }\end{array}$ & $\begin{array}{l}\text { Tropical } \\
\text { America }\end{array}$ & Herb & Occasional \\
\hline
\end{tabular}


Nandita Sarma et al. 329

\begin{tabular}{|c|c|c|c|c|c|}
\hline $\begin{array}{c}\text { Species name with collection } \\
\text { number }\end{array}$ & $\begin{array}{l}\text { Flowers } \\
\text { \& fruits }\end{array}$ & $\begin{array}{l}\text { Local name in } \\
\text { Assamese }\end{array}$ & Nativity & Habit & Occurrence \\
\hline $\begin{array}{l}\text { Blumea lacera (Burm.f.) DC. } \\
\text { [Compositae]; N. Sarma } 136579 \\
\text { (ASSAM) }\end{array}$ & $\begin{array}{l}\text { August - } \\
\text { February }\end{array}$ & $\begin{array}{l}\text { Kukur- } \\
\text { shuta/Kukur- } \\
\text { sunga }\end{array}$ & $\begin{array}{l}\text { Tropical } \\
\text { America }\end{array}$ & Herb & Frequent \\
\hline $\begin{array}{l}\text { Calotropis gigantea }(\mathrm{L} .) \\
\text { Dryand. [Apocynaceae]; } N \text {. } \\
\text { Sarma } 136373 \text { (ASSAM) }\end{array}$ & $\begin{array}{l}\text { Througho } \\
\text { ut the year }\end{array}$ & Akan & $\begin{array}{l}\text { Tropical } \\
\text { Africa }\end{array}$ & Shrub & Occasional \\
\hline $\begin{array}{l}\text { Cardamine hirsuta L. } \\
\text { [Brassicaceae]; N. Sarma } \\
136578 \text { (ASSAM) }\end{array}$ & $\begin{array}{l}\text { July - } \\
\text { February }\end{array}$ & - & $\begin{array}{l}\text { Tropical } \\
\text { America }\end{array}$ & Herb & Occasional \\
\hline $\begin{array}{l}\text { Chenopodium album L. } \\
\text { [Amaranthaceae]; N. Sarma } \\
136577 \text { (ASSAM) }\end{array}$ & & Jilmil & Mexico & Herb & Rare \\
\hline $\begin{array}{l}\text { Chromolaena odorata (L.) R.M. } \\
\text { King \& H. Rob. [Compositae]; } \\
\text { N. Sarma } 133898 \text { (ASSAM) }\end{array}$ & $\begin{array}{l}\text { December } \\
\text { - May }\end{array}$ & Germani bon & $\begin{array}{l}\text { Tropical } \\
\text { America }\end{array}$ & Shrub & Dominant \\
\hline $\begin{array}{l}\text { Cleome gynandra L. } \\
\text { [Cleomaceae]; N. Sarma } 136576 \\
\text { (ASSAM) }\end{array}$ & $\begin{array}{l}\text { September } \\
- \\
\text { December }\end{array}$ & Bhutmala & $\begin{array}{l}\text { Tropical } \\
\text { America }\end{array}$ & Herb & Rare \\
\hline $\begin{array}{l}\text { Cleome rutidosperma } \text { DC. } \\
\text { [Cleomaceae]; N. Sarma } 134062 \\
\text { (ASSAM) }\end{array}$ & $\begin{array}{l}\text { August - } \\
\text { November }\end{array}$ & - & $\begin{array}{l}\text { Tropical } \\
\text { America }\end{array}$ & Herb & Occasional \\
\hline $\begin{array}{l}\text { Cleome viscosa } \mathrm{L} \text {. [Cleomaceae]; } \\
\text { N. Sarma } 136435 \text { (ASSAM) }\end{array}$ & $\begin{array}{l}\text { August - } \\
\text { December }\end{array}$ & Hurhuria & $\begin{array}{l}\text { Tropical } \\
\text { America }\end{array}$ & Herb & Rare \\
\hline $\begin{array}{l}\text { Crotalaria pallida Aiton } \\
\text { [Fabaceae]; N. Sarma } 134008 \\
\text { (ASSAM) }\end{array}$ & $\begin{array}{l}\text { August - } \\
\text { March }\end{array}$ & Jhun-jhuniya & $\begin{array}{l}\text { Tropical } \\
\text { America }\end{array}$ & Herb & Rare \\
\hline $\begin{array}{l}\text { Croton bonplandianus Baill. } \\
\text { [Euphorbiaceae]; N. Sarma } \\
136302 \text { (ASSAM) }\end{array}$ & $\begin{array}{l}\text { August - } \\
\text { December }\end{array}$ & Bon-tulsi & $\begin{array}{l}\text { Temperate } \\
\text { South } \\
\text { America }\end{array}$ & Herb & Rare \\
\hline $\begin{array}{l}\text { Cuscuta reflexa } \text { Roxb. } \\
\text { [Convolvulaceae]; N. Sarma } \\
134033 \text { (ASSAM) }\end{array}$ & $\begin{array}{l}\text { August - } \\
\text { December }\end{array}$ & Akashi-lata & $\begin{array}{l}\text { Mediterrane } \\
\text { an }\end{array}$ & Climber & Occasional \\
\hline $\begin{array}{l}\text { Cyperus difformis L. } \\
\text { [Cyperaceae]; N. Sarma } 136575 \\
\text { (ASSAM) }\end{array}$ & $\begin{array}{l}\text { August - } \\
\text { December }\end{array}$ & - & $\begin{array}{l}\text { Tropical } \\
\text { America }\end{array}$ & Herb & Rare \\
\hline $\begin{array}{l}\text { Cyperus iria L. [Cyperaceae]; } N \text {. } \\
\text { Sarma } 136574 \text { (ASSAM) }\end{array}$ & $\begin{array}{l}\text { August - } \\
\text { January }\end{array}$ & - & $\begin{array}{l}\text { Tropical } \\
\text { America }\end{array}$ & Herb & Rare \\
\hline $\begin{array}{l}\text { Datura metel L. [Solanaceae]; } \\
\text { N. Sarma } 136573 \text { (ASSAM) }\end{array}$ & $\begin{array}{l}\text { August - } \\
\text { February }\end{array}$ & Kala-dhatura & $\begin{array}{l}\text { Tropical } \\
\text { America }\end{array}$ & Shrub & Rare \\
\hline $\begin{array}{l}\text { Echinochloa colona } \text { (L.) Link } \\
\text { [Poaceae]; N. Sarma } 136572 \\
\text { (ASSAM) }\end{array}$ & $\begin{array}{l}\text { August - } \\
\text { March }\end{array}$ & Binoi-bon & $\begin{array}{l}\text { Tropical } \\
\text { South } \\
\text { America }\end{array}$ & Herb & Occasional \\
\hline
\end{tabular}


330 Invasive flora of Amchang Wildlife Sanctuary

\begin{tabular}{|c|c|c|c|c|c|}
\hline $\begin{array}{c}\text { Species name with collection } \\
\text { number }\end{array}$ & $\begin{array}{l}\text { Flowers } \\
\text { \& fruits }\end{array}$ & $\begin{array}{l}\text { Local name in } \\
\text { Assamese }\end{array}$ & Nativity & Habit & Occurrence \\
\hline $\begin{array}{l}\text { Eclipta prostrata }(\mathrm{L} .) \mathrm{L} . \\
\text { [Compositae]; N. Sarma } 136571 \\
\text { (ASSAM) }\end{array}$ & $\begin{array}{l}\text { June - } \\
\text { March }\end{array}$ & Keharaj & $\begin{array}{l}\text { Tropical } \\
\text { America }\end{array}$ & Herb & Rare \\
\hline $\begin{array}{l}\text { Eichhornia crassipes (Mart.) } \\
\text { Solms [Pontederiaceae]; } N \text {. } \\
\text { Sarma } 136570 \text { (ASSAM) }\end{array}$ & $\begin{array}{l}\text { August - } \\
\text { December }\end{array}$ & Pani meteka & $\begin{array}{l}\text { Tropical } \\
\text { America }\end{array}$ & $\begin{array}{l}\text { Aquatic } \\
\text { herb }\end{array}$ & Frequent \\
\hline $\begin{array}{l}\text { Euphorbia hirta } \text { L. } \\
\text { [Euphorbiaceae]; N. Sarma } \\
136569 \text { (ASSAM) }\end{array}$ & $\begin{array}{l}\text { August- } \\
\text { December }\end{array}$ & Gakhirati bon & $\begin{array}{l}\text { Tropical } \\
\text { America }\end{array}$ & Herb & Rare \\
\hline $\begin{array}{l}\text { Evolvulus nummularius (L.) L. } \\
\text { [Convolvulaceae]; N. Sarma } \\
136347 \text { (ASSAM) }\end{array}$ & $\begin{array}{l}\text { October - } \\
\text { February }\end{array}$ & Bhui- ankra & $\begin{array}{l}\text { Tropical } \\
\text { America }\end{array}$ & Herb & Occasional \\
\hline $\begin{array}{l}\text { Galinsoga parviflora Cav. } \\
\text { [Compositae]; N. Sarma } 136568 \\
\text { (ASSAM) }\end{array}$ & $\begin{array}{l}\text { August - } \\
\text { January }\end{array}$ & $\begin{array}{l}\text { Potato weed } \\
\text { (E) }\end{array}$ & $\begin{array}{l}\text { Tropical } \\
\text { America }\end{array}$ & Herb & Rare \\
\hline $\begin{array}{l}\text { Hyptis suaveolens (L.) Poit. } \\
\text { [Lamiaceae]; N. Sarma } 134016 \\
\text { (ASSAM) }\end{array}$ & $\begin{array}{l}\text { August - } \\
\text { March }\end{array}$ & Ukma & $\begin{array}{l}\text { Tropical } \\
\text { America }\end{array}$ & Herb & Rare \\
\hline $\begin{array}{l}\text { Impatiens balsamina } \mathrm{L} . \\
\text { [Balsaminaceae]; N. Sarma } \\
136567 \text { (ASSAM) }\end{array}$ & $\begin{array}{l}\text { July - } \\
\text { January }\end{array}$ & Keruphul & $\begin{array}{l}\text { Tropical } \\
\text { America }\end{array}$ & Herb & Occasional \\
\hline $\begin{array}{l}\text { Imperata cylindrica (L.) } \\
\text { Raeusch. [Poaceae]; N. Sarma } \\
134086 \text { (ASSAM) }\end{array}$ & $\begin{array}{l}\text { July- } \\
\text { December }\end{array}$ & Ulu-bon & $\begin{array}{l}\text { Tropical } \\
\text { America }\end{array}$ & Herb & Rare \\
\hline $\begin{array}{l}\text { Ipomoea hederifolia } \text { L. } \\
\text { [Convolvulaceae]; N. Sarma } \\
134063 \text { (ASSAM) }\end{array}$ & $\begin{array}{l}\text { August - } \\
\text { December }\end{array}$ & - & $\begin{array}{l}\text { Tropical } \\
\text { America }\end{array}$ & Climber & Rare \\
\hline $\begin{array}{l}\text { Lantana camara L. } \\
\text { [Verbenaceae]; N. Sarma } \\
133991 \text { (ASSAM) }\end{array}$ & $\begin{array}{l}\text { Througho } \\
\text { ut the year }\end{array}$ & Goo-phul & $\begin{array}{l}\text { Tropical } \\
\text { America }\end{array}$ & Shrub & Dominant \\
\hline $\begin{array}{l}\text { Ludwigia octovalvis (Jacq.) P.H. } \\
\text { Raven [Onagraceae]; N. Sarma } \\
133809 \text { (ASSAM) }\end{array}$ & $\begin{array}{l}\text { July- } \\
\text { December }\end{array}$ & Bon-jalakia & $\begin{array}{l}\text { Tropical } \\
\text { Africa }\end{array}$ & Herb & Frequent \\
\hline $\begin{array}{l}\text { Mikania micrantha Kunth } \\
{[\text { Compositae }] ; \text { N. Sarma } 133839} \\
\text { (ASSAM) }\end{array}$ & $\begin{array}{l}\text { December } \\
\text { - April }\end{array}$ & Gahori-lata & $\begin{array}{l}\text { Tropical } \\
\text { America }\end{array}$ & Climber & Dominant \\
\hline $\begin{array}{l}\text { Mimosa pudica L. [Fabaceae]; } \\
\text { N. Sarma } 136434 \text { (ASSAM) }\end{array}$ & $\begin{array}{l}\text { July - } \\
\text { February }\end{array}$ & Lajukilota & Brazil & Herb & Frequent \\
\hline $\begin{array}{l}\text { Monochoria vaginalis (Burm.f.) } \\
\text { C.Presl [Pontederiaceae]; } N \text {. } \\
\text { Sarma } 134025 \text { (ASSAM) }\end{array}$ & $\begin{array}{l}\text { August - } \\
\text { December }\end{array}$ & Bhat meteka & $\begin{array}{l}\text { Tropical } \\
\text { America }\end{array}$ & $\begin{array}{l}\text { Aquatic } \\
\text { herb }\end{array}$ & Occasional \\
\hline $\begin{array}{l}\text { Nicotiana plumbaginifolia Viv. } \\
\text { [Solanaceae]; N. Sarma } 136566 \\
\text { (ASSAM) }\end{array}$ & $\begin{array}{l}\text { December } \\
\text { - April }\end{array}$ & Ulu-lai/ dhopat & $\begin{array}{l}\text { Tropical } \\
\text { America }\end{array}$ & Herb & Rare \\
\hline $\begin{array}{l}\text { Ocimum americanum L. } \\
\text { [Lamiaceae]; N. Sarma } 133988 \\
\text { (ASSAM) }\end{array}$ & $\begin{array}{l}\text { August - } \\
\text { January }\end{array}$ & Bon-tulsi & $\begin{array}{l}\text { Tropical } \\
\text { America }\end{array}$ & Shrub & Rare \\
\hline $\begin{array}{l}\text { Oxalis corniculata } \mathrm{L} . \\
\text { [Oxalidaceae]; N. Sarma } 136483 \\
\text { (ASSAM) }\end{array}$ & $\begin{array}{l}\text { August - } \\
\text { December }\end{array}$ & Saru-tengesi & Europe & Herb & Occasional \\
\hline
\end{tabular}


Nandita Sarma et al. 331

\begin{tabular}{|c|c|c|c|c|c|}
\hline $\begin{array}{c}\text { Species name with collection } \\
\text { number }\end{array}$ & $\begin{array}{l}\text { Flowers } \\
\text { \& fruits }\end{array}$ & $\begin{array}{l}\text { Local name in } \\
\text { Assamese }\end{array}$ & Nativity & Habit & Occurrence \\
\hline $\begin{array}{l}\text { Parthenium hysterophorus L. } \\
\text { [Compositae]; N. Sarma } 136407 \\
\text { (ASSAM) }\end{array}$ & $\begin{array}{l}\text { July - } \\
\text { February }\end{array}$ & Gajar ghass & $\begin{array}{l}\text { Tropical } \\
\text { North } \\
\text { America }\end{array}$ & Herb & Dominant \\
\hline $\begin{array}{l}\text { Passiflora foetida } \text { L. } \\
\text { [Passifloraceae]; N. Sarma } \\
136441 \text { (ASSAM) }\end{array}$ & $\begin{array}{l}\text { June - } \\
\text { December }\end{array}$ & Junuka phul & $\begin{array}{l}\text { Tropical } \\
\text { South } \\
\text { America }\end{array}$ & Climber & Rare \\
\hline $\begin{array}{l}\text { Peperomia pellucida }(\text { L.) Kunth } \\
\text { [Piperaceae]; N. Sarma } 136587 \\
\text { (ASSAM) }\end{array}$ & $\begin{array}{l}\text { July- } \\
\text { December }\end{array}$ & Pononua & $\begin{array}{l}\text { Tropical } \\
\text { South } \\
\text { America }\end{array}$ & Herb & Occasional \\
\hline $\begin{array}{l}\text { Physalis minima L. [Solanaceae]; } \\
\text { N. Sarma } 136565 \text { (ASSAM) }\end{array}$ & $\begin{array}{l}\text { July - } \\
\text { January }\end{array}$ & Kapalfuta & $\begin{array}{l}\text { Tropical } \\
\text { America }\end{array}$ & Herb & Rare \\
\hline $\begin{array}{l}\text { Saccharum spontaneum } \text { L. } \\
\text { [Poaceae]; N. Sarma } 136513 \\
\text { (ASSAM) }\end{array}$ & $\begin{array}{l}\text { August - } \\
\text { December }\end{array}$ & Kahua-bon & $\begin{array}{l}\text { Tropical } \\
\text { West Asia }\end{array}$ & Herb & Rare \\
\hline $\begin{array}{l}\text { Scoparia dulcis L. } \\
\text { [Plantaginaceae]; N. Sarma } \\
136446 \text { (ASSAM) }\end{array}$ & $\begin{array}{l}\text { July - } \\
\text { December }\end{array}$ & Seni bon & $\begin{array}{l}\text { Tropical } \\
\text { America }\end{array}$ & Herb & Frequent \\
\hline $\begin{array}{l}\text { Senna alata (L.) Roxb. [Fabaceae } \\
\text { N. Sarma } 133907 \text { (ASSAM) }\end{array}$ & $\begin{array}{l}\text { May - } \\
\text { December }\end{array}$ & Khor-goch & West indies & Shrub & Occasional \\
\hline $\begin{array}{l}\text { Senna occidentalis }(\text { L.) Link } \\
\text { [Fabaceae]; N. Sarma } 136564 \\
\text { (ASSAM) }\end{array}$ & $\begin{array}{l}\text { July - } \\
\text { December }\end{array}$ & Hantthenga & $\begin{array}{l}\text { Tropical } \\
\text { South } \\
\text { America }\end{array}$ & Herb & Rare \\
\hline $\begin{array}{l}\text { Senna tora (L.) Roxb. [Fabaceae]; } \\
\text { N. Sarma } 136563 \text { (ASSAM) }\end{array}$ & $\begin{array}{l}\text { August - } \\
\text { December }\end{array}$ & Medeluwa & $\begin{array}{l}\text { Tropical S } \\
\text { America }\end{array}$ & Herb & Rare \\
\hline $\begin{array}{l}\text { Sida acuta } \text { Burm.f. [Malvaceae]; } \\
\text { N. Sarma } 133806 \text { (ASSAM) }\end{array}$ & $\begin{array}{l}\text { July - } \\
\text { December }\end{array}$ & Boriala & $\begin{array}{l}\text { Tropical } \\
\text { America }\end{array}$ & Herb & Occasional \\
\hline $\begin{array}{l}\text { Solanum americanum Mill. } \\
\text { [Solanaceae]; N. Sarma } 136561 \\
\text { (ASSAM) }\end{array}$ & $\begin{array}{l}\text { June }- \\
\text { December }\end{array}$ & Laskosi/ koisi & $\begin{array}{l}\text { Tropical } \\
\text { America }\end{array}$ & Herb & Rare \\
\hline $\begin{array}{l}\text { Solanum torvum Sw.[Solanaceae] } \\
\text { N. Sarma } 136339 \text { (ASSAM) }\end{array}$ & $\begin{array}{l}\text { October- } \\
\text { March }\end{array}$ & Hati bhekuri & West Indies & Shrub & Rare \\
\hline $\begin{array}{l}\text { Solanum viarum Dunal } \\
\text { [Solanaceae]; N. Sarma } 136560 \\
\text { (ASSAM) }\end{array}$ & $\begin{array}{l}\text { August - } \\
\text { February }\end{array}$ & - & $\begin{array}{l}\text { Tropical } \\
\text { America }\end{array}$ & Herb & Rare \\
\hline $\begin{array}{l}\text { Stachytarpheta jamaicensis (L.) } \\
\text { Vahl [Verbenaceae]; N. Sarma } \\
133889 \text { (ASSAM) }\end{array}$ & $\begin{array}{l}\text { Mainly in } \\
\text { rainy } \\
\text { season }\end{array}$ & - & $\begin{array}{l}\text { Tropical } \\
\text { America }\end{array}$ & Herb & Rare \\
\hline $\begin{array}{l}\text { Synedrella nodiflora (L.) } \\
\text { Gaertn. [Compositae]; N. Sarma } \\
133830 \text { (ASSAM) }\end{array}$ & $\begin{array}{l}\text { August - } \\
\text { January }\end{array}$ & Suhani ban & West Indies & Herb & Rare \\
\hline $\begin{array}{l}\text { Tridax procumbens (L.) L. } \\
\text { [Compositae]; N. Sarma } 134087 \\
\text { (ASSAM) }\end{array}$ & $\begin{array}{l}\text { Througho } \\
\text { ut the year }\end{array}$ & $\begin{array}{l}\text { Mexican } \\
\text { Daisy(E) }\end{array}$ & $\begin{array}{l}\text { Tropical } \\
\text { Central } \\
\text { America }\end{array}$ & Herb & Frequent \\
\hline $\begin{array}{l}\text { Triumfetta rhomboidea Jacq. } \\
\text { [Malvaceae]; N. Sarma } 136559 \\
\text { (ASSAM) }\end{array}$ & $\begin{array}{l}\text { June }- \\
\text { December }\end{array}$ & Agora & $\begin{array}{l}\text { Tropical } \\
\text { America }\end{array}$ & Herb & Rare \\
\hline $\begin{array}{l}\text { Urena lobata L. [Malvaceae]; } N \text {. } \\
\text { Sarma } 133832 \text { (ASSAM) }\end{array}$ & $\begin{array}{l}\text { July - } \\
\text { December }\end{array}$ & Honborolua & $\begin{array}{l}\text { Tropical } \\
\text { Africa }\end{array}$ & $\begin{array}{l}\text { Shrub } \\
\text { (annual) }\end{array}$ & Rare \\
\hline $\begin{array}{l}\text { Xanthium strumarium L. } \\
\text { [Compositae]; N. Sarma } 136558 \\
\text { (ASSAM) }\end{array}$ & $\begin{array}{l}\text { August - } \\
\text { March }\end{array}$ & Aagora-kata & $\begin{array}{l}\text { Tropical } \\
\text { America }\end{array}$ & Herb & Rare \\
\hline
\end{tabular}


The frequency of occurrence of species showed that the most species were of rare occurrence ( 29 species) followed by occasional (14 species), frequent ( 7 species) and dominant (7 species) occurrences (Table 1). The dominant species included: Ageratum conyzoides, Alternanthera philoxeroides, Alternanthera sessilis, Chromolaena odorata, Lantana camara, Mikania micrantha and Parthenium hysterophorus. The habit of the species showed that the most species were herbs ( 42 species) followed by shrubs ( 8 species) and undershrub (1 species), and four climbers (Cuscuta reflexa, Ipomoea hederifolia, Mikania micrantha and Passiflora foetida) besides two aquatic herbs (Eichhornia crassipes and Monochoria vaginalis) (Table 1). Most of the invasive species were from North and South America (48 species), Tropical Africa (3 species, Calotropis gigantea, Ludwigia octovalvis, Urena lobata), West Indies (3 species, Senna

Table 2. A list of invasive plants used as local medicine by the local people in Amchang Wildlife Sanctuary.

\begin{tabular}{|c|c|c|}
\hline Species name & Parts used & Purpose \\
\hline Ageratum conyzoides & Leaf & Fresh leaf juice applied in cuts \\
\hline Alternanthera sessilis & Tender shoots & Tender shoots are eaten for curing dysentery \\
\hline Amaranthus spinosus & Whole plant & The paste of whole plant is applied on eczema \\
\hline Argemone mexicana & Leaf & Leaves applied locally in skin disease \\
\hline Chromolaena odorata & Leaf & Leaf paste is applied locally in toothache \\
\hline Croton bonplandianus & Leaf & Leaf extract is used in cataract eye \\
\hline Cuscuta reflexa & Stem & Extracted juice is given to cure jaundice \\
\hline Datura metel & Leaf & $\begin{array}{l}\text { Fresh leaves are boiled in coconut oil and the oil is } \\
\text { externally applied as an antiseptic }\end{array}$ \\
\hline Eclipta prostrata & Leaf & Extract mix with oil for hair growth \\
\hline Euphorbia hirta & Leaf & Leaf paste is used in wounds and poisonous bites \\
\hline Evolvulus nummularius & Leaf, Stem & $\begin{array}{l}\text { Plant paste applied externally to reduce tonsillitis } \\
\text { pain }\end{array}$ \\
\hline Lantana camara & Leaf & $\begin{array}{l}\text { Leaf paste is used in various skin diseases and also } \\
\text { to stop bleeding }\end{array}$ \\
\hline Mikania micrantha & Leaves & The crushed leaves are used in minor cuts \\
\hline Mimosa pudica & Root & $\begin{array}{l}\text { Root paste is applied on septic ulcer for quick } \\
\text { healing }\end{array}$ \\
\hline Monochoria vaginalis & Rhizome & $\begin{array}{l}\text { Rhizome is eaten regularly while empty stomach to } \\
\text { cure asthmatic trouble }\end{array}$ \\
\hline Ocimum americanum & Leaf & Leaf paste is externally applied to treat itching \\
\hline Oxalis corniculata & Leaf & Leaf juice is applied to cure scabies \\
\hline Peperomia pellucida & Leaf, Stem & $\begin{array}{l}\text { Plant paste externally to reduce pimple and white } \\
\text { spots of the body }\end{array}$ \\
\hline Senna alata & Leaf & Leaf juice is applied on ringworm \\
\hline Senna occidentalis & Leaf & $\begin{array}{l}\text { Leaf powder is made into a paste applied to cure } \\
\text { skin diseases }\end{array}$ \\
\hline Senna tora & Leaf & Leaf paste is applied on scabies and ringworm \\
\hline Sida acuta & Leaf & The leaf paste is layered on the boils \\
\hline Tridax procumbens & Leaf & The leaf paste is used in cellulites \\
\hline Urena lobata & Seed & $\begin{array}{l}\text { Seeds are crushed into a paste and applied on } \\
\text { scabies and ringworm }\end{array}$ \\
\hline
\end{tabular}

alata, Solanum torvum, Synedrella nodiflora), Europe (1 species, Oxalis corniculata), Tropical West Asia (1 species, Saccharum spontaneum) and Mediterranean (1 species, Cuscuta reflexa).

The local inhabitants in and around the sanctuary have learned the use of a number of these species for curing various ailments. In most cases the leaf of the plant is used and in 
Table 3. A list of invasive plants used as vegetable by the local people in Amchang Wildlife Sanctuary.

\begin{tabular}{|l|l|l|}
\hline \multicolumn{1}{|c|}{ Species name } & \multicolumn{1}{c|}{ Parts used } & \multicolumn{1}{c|}{ Mode of use } \\
\hline Alternanthera philoxeroides & Tender shoot & Leaves cooked as vegetable \\
\hline Alternanthera sessilis & Tender shoot & Leaves cooked as vegetable \\
\hline Amaranthus spinosus & $\begin{array}{l}\text { Tender shoots } \\
\text { and leaves }\end{array}$ & Stem and leaves cooked as vegetable \\
\hline Bidens pilosa & Leaves & Young leaves and shoots cooked as vegetable \\
\hline Chenopodium album & $\begin{array}{l}\text { Young shoot/ } \\
\text { Leaves }\end{array}$ & Tender shoots fried and eaten \\
\hline Euphorbia hirta & $\begin{array}{l}\text { Young shoots } \\
\text { and leaves }\end{array}$ & $\begin{array}{l}\text { Young shoots and leaves are used as mixed } \\
\text { vegetable }\end{array}$ \\
\hline Oxalis corniculata & Leaves/Stem & $\begin{array}{l}\text { Young shoots and leaves are used as } \\
\text { vegetable }\end{array}$ \\
\hline
\end{tabular}

some cases stem, tender shoots, roots, rhizome and seeds are used. Most of the applications are of the leaf paste or leaf juice for curing skin related diseases followed by the cure of stomach related ailments (such as dysentery), fever, pain and antidote to poison (Table 2).

A variety of species are also used as vegetable by the local inhabitants in and around Amchang wildlife sanctuary. In most cases the leaf of the plant or tender shoot is used as vegetable (Table 3). These are used raw or boiled or fried in isolation or in mix with other vegetables such as potato and onion. The local people believe that the use of some of these plants as vegetable also renders medicinal efficacy in improving their immunity.

\section{CONCLUSIONS}

In Amchang Wildlife Sanctuary, a large number of invasive plant species occur in local flora. The invasive species grow quickly and germinate in wide range of physical conditions. These perennial species can flower in large quantities, produce many seeds, and disperse seeds effectively to occupy newer habitats. Our observations in Amchang Wildlife Sanctuary have shown that an invasion of Parthenium hysterophorus was seen from 2014 onwards and this species spread in a few months' time in considerable area. The native aquatic species were rapidly replaced by the exponentially growing Eichhornia crassipes and Lantana camara as dense thickets. Mikania micrantha has grown to the level of menace on the branches of trees and shrubs. The rapid proliferation of invasive species in roadsides under the forest canopy not only poses a threat to the forest flora and community composition, but also alters biomass and nutrient dynamics of the ecosystem. The presence of the recorded invasive plants in this study poses a serious challenge for their control in Amchang Wildlife Sanctuary.

\section{Acknowledgements}

The authors are thankful to the officials of the Department of Forests and Environment, Government of Assam for granting permission to work in Amchang Wildlife Sanctuary, the authorities of the Botanical Survey of India, and the Head, Department of Botany, North Eastern Hill University, Shillong for laboratory facilities. The forest guides and Assam Protection Forest Force rendered help in carrying out the field work. The local people of the area and vegetable vendors are thanked for their kind assistance and useful information. One of the authors (US) is studying the patterns in the forests and the flora of northeastern region of India under the doctor of science programme at the Department of Botany, NorthEastern Hill University, Shillong and acknowledges partial support from a networking project 
grant (NMHS-2017/LG-01/475 dated 22.12.2017) on "Invasive Alien Plants in Himalayas: Status, Ecological Impact and Management" which has been sanctioned to Prof. R.K. Kohli (Project Coordinator) of Central University of Punjab, Bathinda by the National Mission on Himalayan Studies (NMHS) of the G.B. Pant National Institute of Himalayan Environment and Sustainable Development, Kosi-Katarmal, Almora.

\section{LITERATURE CITED}

Anonymous 2019. The Official State Portal of Assam. https://assam.gov.in/en/main/ Wildlife\%20Sancturies. Accessed on: 24 Dec 2019.

Barooah, C. \& Ahmed, I. 2014. Plant diversity of Assam-A checklist of Angiosperms \& Gymnosperms. Assam Science Technology and Environment Council, Assam.

Bhatt, J.R.; Singh, J.S.; Singh, S.P.; Tripathi, R.S. \& Kohli R.K. (eds.) 2011. Invasive alien plants: an ecological appraisal for the Indian subcontinent. CABI, UK.

Boro, A. \& Sarma, G. C. 2013. A check list of exotic plants in the Bornadi Wildlife Sanctuary in Assam, India. Pleione 7(1): 46 - 51.

Buragohai, S. 2007. Exotic plants of Guwahati, Assam, India. Ph.D Thesis, University of Gauhati, Guwahati.

Das, A.P. 2002: Survey of naturalised exotics in the flora of Darjiling Hills, West Bengal, (India). J. Econ. Tax. Bot., 26(1): 31 - 37.

Das, K. \& Duarah, P. 2013. Invasive Alien Plant Species in the Roadside Areas of Jorhat, Assam: Their Harmful Effects and Beneficial Uses. Intn. J. Engn. Res. Applic. 3(5): $353-358$.

Hooker, J.D. 1872-97. Flora of British India. Vols. 1 through 7. L. Reeve and Co., Ashford, Kent, London.

Jain, S.K. \& Rao, R.R. 1977. A Hand Book of Field and Herbarium Technique. Today \& Tomorrow's Publication, New Delhi.

Kanjilal, U.N., Kanjilal, P.C. \& Das, A. 1936. Flora of Assam, Vol. 2. Government of Assam, Shillong.

Kanjilal, U.N., Kanjilal, P.C.; Das, A. \& De, R.N. 1938. Flora of Assam, Vol. 3. Government of Assam, Shillong.

Kanjilal, U.N.; Kanjilal, P.C.; Das, A. \& Purkayastha, C. 1934. Flora of Assam, Vol. 1. Government of Assam, Shillong.

Kanjilal, U.N.; Kanjilal, P.C.; De, R.N. \& Das, A. 1940. Flora of Assam, Vol. 4. Government of Assam, Shillong.

Khuroo, A.A.; Rashid, I.; Reshi, Z.; Dar, G.H. \& Wafai, B.A. 2007. The alien ûora of Kashmir Himalaya. Biological Invasions 9: 269 - 292.

Majumdar, K., Shankar, U. \& Datta, B.K. 2014. Lower altitudinal variation in habitat associations, tree diversity and co-dominant population structures along moist deciduous forests dominated by sal (Shorea robusta) in Northeast India. For. Sci. Tech. 10(4): 201 - 212. (DOI: 10.1080/21580103.2014.920732)

Myers, N. 2003. Biodiversity hotspots revisited. BioScience 53: 916 - 917.

Reddy, C.S.; Bagyanarayana, G.; Reddy, K.N. \& Raju, V.S. 2008. Invasive alien flora of India. National Biological Information Infrastructure, US Geological Survey, USA. 
Sekar, K.C. 2012. Invasive Alien Plants of Indian Himalayan Region- Diversity and Implication. Amer. J. Pl. Sci. 3: $177-184$.

Shankar, U. 1991. Nutrient analysis in degraded ecosystems (grasslands) of Meghalaya. Ph.D. Thesis, North-Eastern Hill University, Shillong.

Shankar, U. 2001. A case of high tree diversity in a 'sal' (Shorea robusta)-dominated lowland forest of eastern Himalaya: floristic composition, regeneration and conservation. Curr. Sci. 81(7): $776-786$.

Shankar, U. 2017. Yes, we have tropical rainforest far from ehe Wquator in Meghalaya. The NEHU J. 15(1): 1 - 9.

Shankar, U. 2019. Phytosociology of stratification in a lowland tropical rainforest occurring north of the tropic of cancer in Meghalaya, India, Pl. Diver. 41: 295 - 299.

Shankar, U. \& Tripathi, A.K. 2017. Rainforests north of the Tropic of Cancer: physiognomy, floristics and diversity in 'lowland rainforests' of Meghalaya, India. Pl. Diver. 39: $20-36$.

Shankar, U.; Tripathi, R.S. \& Pandey, H.N. 1991. Structure and seasonal dynamics of humid tropical grasslands in Meghalaya, India. J. Veget. Sci. 2: $711-714$.

Shankar, U.; Yadava, A.S.; Rai J.P.N. \& Tripathi, R.S. 2011. Status of alien plant invasions in north-eastern region of India. In: Bhatt, J. R., Singh, J. S., Singh, S. P., Tripathi, R.S. \& Kohli, R. K. (eds.), Invasive alien plants: an ecological appraisal for the Indian subcontinent. CABI, UK. Pp. 174 - 188.

The Plant List 2013. The Plant List. Version 1.1. http://www.theplantlist.org/. [Accessed on: December 21, 2019]

Tripathi, A.K. \& Shankar, U. 2014. Patterns of species dominance, diversity and dispersion in 'Khasi hill sal' forest ecosystem in northeast India. For. Ecosys. 1: 1 - 20.

Upadhaya, K. 2015. Structure and floristic composition of subtropical broad-leaved humid forest of Cherrapunji in Meghalaya, northeast India. J Biodiv. Manag. Forestry. 4 (4). https://doi.org/10.4172/2327-4417.1000149.

WFO 2019. World Flora Online. Published on the Internet. http://www.worldfloraonline.org. [Accessed on: 24 Dec. 2019] 